\title{
A rare case of pure primary hemangioma of the scapula: A case report
}

\author{
WEI LI $^{1 *}$, FAN ZOU $^{1 *}$, MIN DAI $^{1}$, BIN ZHANG $^{2}$ and TAO NIE ${ }^{2}$ \\ ${ }^{1}$ Department of Orthopedics; ${ }^{2}$ Multidisciplinary Therapy Center of Musculoskeletal Tumor, \\ The First Affiliated Hospital of Nanchang University, Nanchang, Jiangxi 330006, P.R. China
}

Received July 30, 2014; Accepted May 12, 2015

DOI: 10.3892/ol.2015.3596

\begin{abstract}
Hemangioma is a benign vascular tumor, which may occur in any bone of the body. The most common locations are the spine and craniofacial bone; however, occurrence is extremely rare in the scapula. The current study presented the case of a 58-year-old female, with scapula hemangioma in the left shoulder who presented with joint ache that had lasted for $\sim 1$ year. The main clinical manifestations included local tenderness, an osseous lump and limited shoulder movement with a little pain, which was alleviated by rest. Roentgenogram, computed tomography and magnetic resonance imaging of the left acromion revealed a mass along the inner surface of the scapula of the left shoulder with polycystic expansion and bone destruction. The results of computed tomography and magnetic resonance imaging indicated a pure primary tumor and the lesion was subsequently resected. Notably, the postoperative pathological diagnosis was capillary hemangioma. The aim of the present study was to analyze the clinical and imaging features of scapula hemangioma, which must be considered for the differential diagnosis of scapula tumors. In the present case, no recurrence was identified by X-ray examination 1 year after surgery. The long-term efficacy of surgical treatment requires continuous observation of the patient.
\end{abstract}

\section{Introduction}

Bone hemangiomas are benign lesions originating from new capillaries or cavernous vascular vessels located in the bone. They are characterized by an increased number of normal or abnormal blood vessels. Bone hemangiomas are initially asymptomatic, however, as the tumor grows local pain is often

Correspondence to: Professor Min Dai, Department of Orthopedics, The First Affiliated Hospital of Nanchang University, 17 Yong Wai Zheng Street, Nanchang, Jiangxi 330006, P.R. China E-mail:250132519@qq.com

*Contributed equally

Key words: hemangioma, scapula, misdiagnosis, osteofibrous dysplasia experienced (1). Degradation can occur during the growth of hemangioma (2). These rare, slow growing neoplasms may occur at any age, and account for $\sim 1 \%$ of bone tumors (3-5). Hemangiomas may occur in any bone of the body, most commonly in the spine, craniofacial bone, skull, ribs and long bones (6-15); however, occurrence in the scapula is extremely rare. Hemangiomas of the long bone or flat bone commonly have a 'foam' or 'honeycomb' appearance (16-18). Bone hemangiomas disrupt the cortex and commonly grow expansively, which may result in the lesions being misdiagnosed as aggressive tumors or osteofibrous dysplasia and infectious processes $(9,19)$. At present, treatments include radiotherapy, surgery and vascular embolization (1). In the present study, a rare case of a capillary hemangioma of the scapula, which proved difficult to diagnose, was presented.

To the best of our knowledge, this is the first case of pure primary hemangioma of the scapula that was misdiagnosed as an osteofibrous dysplasia (20) and treated with surgery.

\section{Case report}

In June 2013, a 58 year-old female presented at the First Affiliated Hospital of Nanchang University (Nanchang, China) with intermittent/repeated left shoulder pain, which had lasted for $\sim 1$ year. The main clinical manifestations included local tenderness, an osseous lump and limited shoulder movement with a little pain, which was alleviated by rest. The patient was referred to the Department of Orthopedics for further investigation of local tenderness of the scapula. Written informed consent was obtained from the patient for participation in the present study.

The left shoulder joint was investigated by X-ray examination (Fig. 1A), which revealed a mass with bone destruction of the left acromion. The patient had a previous history of hypertension, which had been successfully managed by ongoing antihypertensive drug treatment. Blood tests revealed that the levels of the tumor markers, carcinoembryonic antigen, cancer antigen 125 and $\alpha$-fetoprotein, were within the normal limits. Three-dimensional reconstruction using $\mathrm{CT}$ revealed swelling of the left shoulder scapula with bone destruction within the soft tissue mass density. Furthermore, interruption and destruction were identified in the cortex and trabecula of the bone, as well as a slight periosteal reaction and visible lesions with separated shadows; however, the mass boundaries were unclear (Fig. 1B). MRI was performed to confirm the 

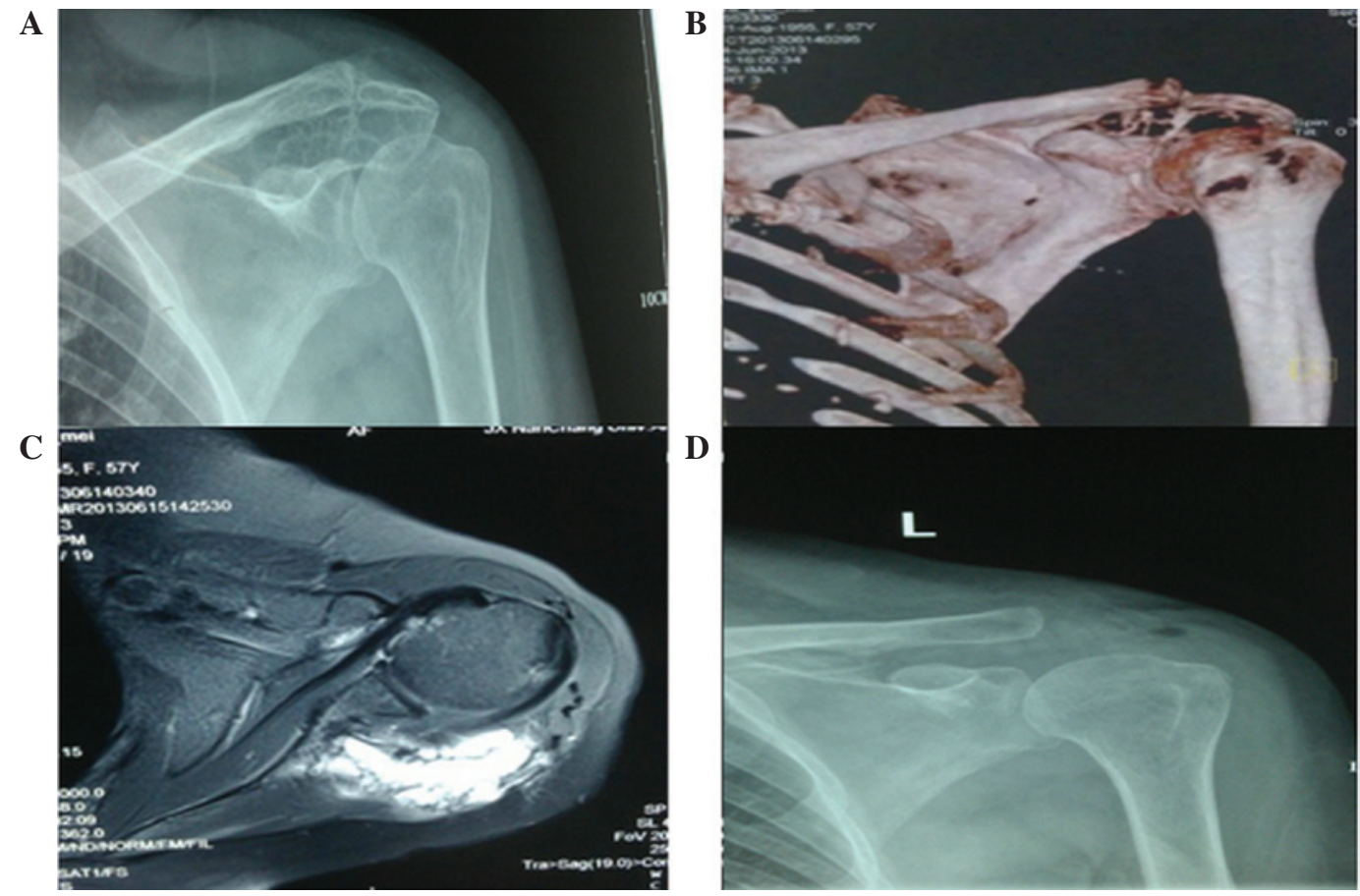

Figure 1. (A) Preoperative X-ray examination showing a mass with bone destruction of the left acromion, suggestive of osteofibrous dysplasia. (B) Preoperative three-dimensional reconstruction of computed tomography examination findings, demonstrating the left shoulder scapula with shadow of abnormal density, suggesting an osteogenic tumor lesion. (C) Preoperative magnetic resonance imaging scan revealing expansive bone destruction of the left scapula acromion with an abnormal signal, suggesting that bone-derived space occupying focus. (D) X-ray examination 1 year after surgery revealing the absence of the left scapula acromion tumor.

A

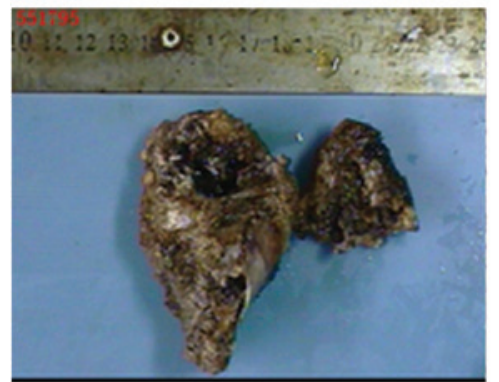

B

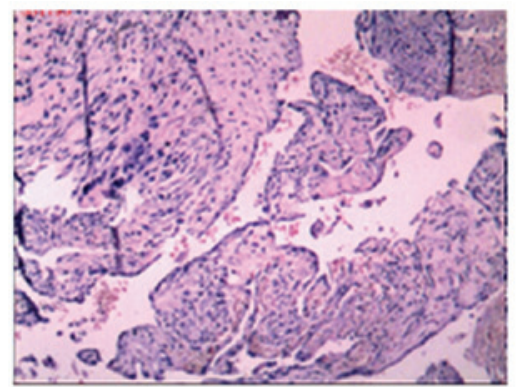

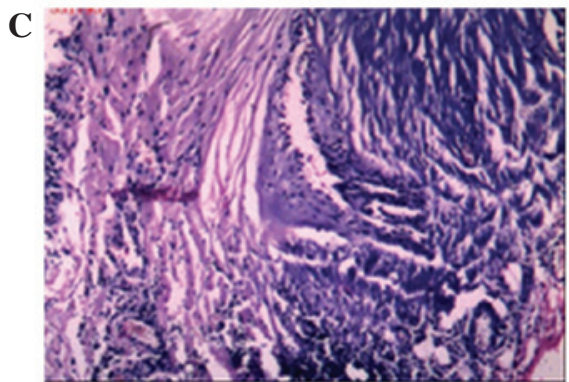

Figure 2. Histological observations. (A) The lesions corresponded to a benign acromion hemangioma originating from scapula. The medullary cavity of two taupe bones enclosed numerous gray-red broken floccules. Light microscopy revealed (B) irregular pipe cavities, exhibiting hemorrhage, thrombosis and recanalization, and (C) a large number of blood vessels, demonstrating hyperplasia between the bone and soft tissue (stain, hematoxylin and eosin; magnification, $\mathrm{x} 100)$.

diagnosis, revealing expansive bone destruction of the left scapula acromion. The mass exhibited longer signal intensity on T2-weighted sequences and equal T1-weighted sequences, while the fat suppression sequences presented as a high signal with a small number of uneven signals. Part of the edge appeared a little fuzzy and the soft tissue around the mass did not present abnormal signal intensity. A number of of small dots distributed around the left humeral neck exhibited long signal intensity on T1- and T2-weighted sequences, and high signals in the fat suppression sequences. Furthermore, a small amount of effusion was observed in the left shoulder joint capsule (Fig. 1C). An MRI scan demonstrated no enlarged lymph nodes or distant metastases.

Notably, a mass along the inner surface of the left shoulder scapula with polycystic expansion and bone destruction was identified, which was diagnosed as osteofibrous dysplasia of the scapula. Subsequently, tumorectomy was performed by bone tumor specialists, since the patient did not present any surgery contraindications. Sterile drapes were disinfected and paved routinely in the right lateral position to expose the operative field following the administration of anesthesia. The left acromion was selected as the center transverse incision, followed by skin, subcutaneous, superficial fascia and scapular muscle incisions. The fascia and soft tissue were stripped to expose the acromion, which exhibited expansive bone destruction within a polycystic intratumor with an uneven surface and rich blood supply. The tumor was excised completely and tissue lesions were removed for pathological examination. A wound drainage tube was inserted and each layer of tissue was sutured to allow complete hemostasis. The estimated blood loss was $200 \mathrm{ml}$ and no blood transfusions were required during surgery. 
Pathological examination revealed two taupe bones with an irregular shape, measuring between $3.5 \times 3.5 \times 1.5 \mathrm{~cm}^{3}$ and $7 \times 5.0 \times 3.2 \mathrm{~cm}^{3}$, and numerous gray-red broken floccules enclosing the medullary cavity. (Fig. 2A) Light microscopy revealed hyperplasia of the blood vessels between the bone and soft tissue (Fig. 2C), with pipe cavities of different sizes and uneven tube walls, exhibiting hemorrhage, thrombosis and recanalization (Fig. 2B). Based on these findings, the tumor was finally diagnosed as a benign left acromion hemangioma originating from the scapula. The patient underwent follow-up X-ray examination 1 year after surgery, which revealed that symptoms had improved and no evidence of recurrence was identified (Fig. 1D).

\section{Discussion}

Hemangioma of the scapula is an uncommon benign vascular tumor, and to the best of our knowledge, no cases have been reported in the literature to date. Thus, the etiology remains unclear. Furthermore, it remains unclear whether all lesions are true neoplasms or should be regarded as hamartomas $(2,21)$. Histologically, four types of hemangioma exist: Capillary, cavernous, venous and mixed. Capillary hemangiomas consist of numerous tortuous small vascular channels lined with epithelium, while the more common cavernous hemangiomas present large dilated vessels lined with a single layer of endothelial cells surrounded by a fibrous stromal layer (22). In general, hemangiomas have a good prognosis with a low recurrence rate $(23,24)$. Although capillary hemangioma of the scapula is extremely rare, it should be considered in the differential diagnosis of scapula tumors. The differential diagnosis may include malignant scapula tumors, such as chondrosarcoma, Ewing's sarcoma, and myeloma or benign scapula tumors, including fibrous dysplasia, osteochondroma, eosinophilic granuloma and aneurysmal bone cysts (22).

Generally, as degradation may occur during the growth of bone hemangioma, the vascular tissue may be replaced by fibrous tissue and achieve self-healing (1). Therefore, asymptomatic patients must be followed-up, without receiving any therapy temporarily. In addition, patients with symptoms or pathological lesions should be followed up as they have a high risk of fracture (25). However, symptomatic patients should receive surgical resection with intralesional ethanol injection, selective arterial embolization, percutaneous vertebroplasty and radiotherapy treatment. It is important to avoid impairing the tumor during surgery as this may lead to severe hemorrhage (26). Thus, preoperative embolization may be beneficial to avoid intraoperative bleeding (27).

With the exception of hemorrhage, the adverse effects of surgery include incomplete removal and long recovery periods (28). The prognosis depends on tumor location and tumor size. Furthermore, it has been reported that the coracoclavicular ligament is a critical component of the acromioclavicular joint, although each component of the joint provides acromioclavicular joint stability in different directions (29). Partial resection, which leads to the destruction of the acromioclavicular joint, does not affect the function of shoulder joint as long as the coracoclavicular ligament is preserved. Surgical excision is the treatment of choice for patients with hemangiomas that have infiltrated the bone and soft tissue, while histological examination confirms diagnosis. In addition, needle biopsy may indicate a definite diagnosis. However, this procedure may be difficult in certain cases, and should be avoided due to the risk of hemorrhage or seeding of the needle tract, unless multiple myeloma or metastatic disease is highly suspected $(30,31)$.

Hemangioma patients are usually asymptomatic and the tumor is often identified incidentally on routine chest roentgenograms. Subsequent CT and MRI scans may reveal the extent of cortical destruction more clearly. The presence of fat density on MRI examinations, well-defined rather than infiltrative borders and sclerotic margins, and a honeycomb appearance are highly suggestive of bone hemangiomas (32-34). Ching et al reported that bone hemangiomas commonly exhibit hypointensity on T1-weighted images and hyper intensity on T2-weighted images depending on the quantity of vascularity and adipose tissue (16). Therefore, it is crucial to evaluate every radiological abnormality carefully and possible accompanying disorders should not be ignored when the diagnosis of the disease is confirmed.

In conclusion, accurate diagnosis is essential for the surgical management of hemangioma. Notably, the majority of bone hemangioma cases are accompanied by slight pain; however, the symptoms of the present case included local tenderness, an osseous lump and limited shoulder movement. As it is difficult to identify such lesions on CT and MRI scans, the present case was misdiagnosed, as rare conditions were not considered. Histological observations may help establish a definitive diagnosis; however, the preoperative clinical manifestations and details of the differential diagnosis were overlooked in the present case. Patient age, tumor location, tumor size, possible pathological type and the other aspects must be considered during preoperative assessment due to the high risk of hemorrhage that is associated with bone hemangioma surgery. Thus, intraoperative precautions must be performed to prevent hemorrhagic shock and other adverse consequences during surgery. In the current study, the patient was misdiagnosed with osteofibrous dysplasia based on the clinical manifestations. The results of X-ray examination indicated a diagnosis of left acromion osteofibrous dysplasia; however, three-dimensional computed tomography (CT) and magnetic resonance imaging (MRI) examinations revealed a mass along the inner surface of the left shoulder scapula, with polycystic expansion and bone destruction. Therefore, clinical examination and careful consideration of patient history are key factors for the identification of diseases, particularly tumors. Further diagnostic pathological examination may clarify the diagnosis (35). However, definitive preoperative diagnosis of hemangioma is of great significance to guide treatment. In the present case, the patient was followed-up for 1 year and has achieved full recovery. The current report analyzed the clinical and imaging features of the scapula hemangioma to highlight possible diagnostic and management methods. The results indicated that careful evaluation of all radiological abnormalities is crucial, to avoid potential diseases being overlooked. Furthermore, this study may increase clinical awareness with regard to hemangioma.

\section{References}

1. Syrimpeis V, Vitsas V and Korovessis P: Lumbar vertebral hemangioma mimicking lateral spinal canal stenosis: Case report and review of literature. J Spinal Cord Med 37: 237-242, 2014. 
2. Hart JL, Edgar MA and Gardner JM: Vascular tumors of bone. Semin Diagn Pathol 31: 30-38, 2014.

3. Shimizu K, Yamashita Y, Hihara J, et al: Cavernous hemangioma of the rib. Ann Thorac Surg 74: 932-934, 2002.

4. Okumura T, Asamura H, Kondo H, Matsuno Y and Tsuchiya R: Hemangioma of the rib: a case report. Jpn J Clin Oncol 30: 354-357, 2000.

5. Gologorsky Y, Shrivastava RK, Panov F, Mascitelli J, Signore AD, Govindaraj S, Smethurst M and Bronster DJ: Primary intraosseous cavernous hemangioma of the clivus: case report and review of the literature. J Neurol Surg Rep 74: 17-22, 2013.

6. Politi M, Romeike BF, Papanagiotou P, et al: Intraosseous hemangioma of the skull with dural tail sign: radiologic features with pathologic correlation. AJNR Am J Neuroradiol 26: 2049-2052, 2005.

7. Schrock WB, Wetzel RJ, Tanner SC and Khan MA: Aggressive hemangioma of the thoracic spine. J Radiol Case Rep 5: 7-13, 2011

8. Tucer B,Ekici MA, Menku A, et al: Surgical management of symptomatic T8 vertebral hemangioma: case report and review of the literature. Turk Neurosurg 23: 680-684, 2013.

9. Oruc M, Atay AE, Karabulut P, et al: An unusual case of cavernous hemangioma of the rib in a young man with lung tuberculosis: a brief review and case report. Intern Med 52: 1263-1265, 2013.

10. Agarwal V, Sreedher G, Weiss KR and Hughes MA: Sacroplasty for symptomatic sacral hemangioma: a novel treatment approach. A case report. Interv Neuroradiol 19: 245-249, 2013.

11. Mridha AR, Kinra P, Sable M, et al: Epithelioid hemangioma of distal femoral epiphysis in a patient with congenital talipes equinovarus. Malays J Pathol 36: 63-66, 2014.

12. Park BH, Hwang E and Kim CH: Primary intraosseous hemangioma in the frontal bone. Arch Plast Surg 40: 283-285, 2013.

13. Atc1 IB, Albayrak S, Yilmaz N, et al: Cavernous hemangioma of the parietal bone. Am J Case Rep 14: 401-404, 2013.

14. Aneja A, Bhattacharyya S, Mydlo J and Inniss S: Testicular seminomatous mixed germ cell tumor with choriocarcinoma and teratoma with secondary somatic malignancy: a case report. J Med Case Rep 8: 1, 2014.

15. Gologorsky Y, Shrivastava RK, Panov F, Mascitelli J, Signore AD, Govindaraj S, Smethurst M and Bronster DJ: Primary intraosseous cavernous hemangioma of the clivus: case report and review of the literature. J Neurol Surg Rep 74: 17-22, 2013.

16. Ching BC, Wong JS, Tan MH and Jara-Lazaro AR: The many faces of intraosseous haemangioma: a diagnostic headache. Singapore Med J 50: e195-e198, 2009.

17. Nakamura H, Kawasaki N, Taguchi M and Kitamura H: Cavernous hemangioma of the rib diagnosed preoperatively by percutaneous needle biopsy. Gen Thorac Cardiovasc Surg 55: 134-137, 2007.

18. Chen KC, Wu CT, Pan CT and Lee YC: Metachronous multiple chest wall osseous hemangiomas. J Thorac Cardiovasc Surg 133: 838-839, 2007.
19. Tew K, Constantine S and Lew WY: Intraosseous hemangioma of the rib mimicking an aggressive chest wall tumor. Diagn Interv Radiol 17: 118-121, 2011.

20. Jung JY, Jee WH, Hong SH, et al: MR findings of the osteofibrous dysplasia. Korean J Radiol 15: 114-122, 2014.

21. Kamala KA, Ashok L and Sujatha GP: Cavernous hemangioma of the tongue: A rare case report. Contemp Clin Dent 5: 95-98, 2014.

22. Gourgiotis S, Piyis A and Panagiotopoulos N: Cavernous hemangioma of the rib: A rare diagnosis. Case Rep Med 2010: 254098,2010

23. Verbeke SL and Bovée JV: Primary vascular tumors of bone: a spectrum of entities? Int J Clin Exp Pathol 4: 541-551, 2011.

24. Mallınson P, Coupal T, Hayes M, et al: Osteosarcoma arising from a haemangioma: case report and review of the literature. Turk Patoloji Derg 30: 137-141, 2014.

25. Abrão FC, Tamagno M, Canzian M, Fernandez Â, Bibas J, Fernandes PM and Jatene FB: Hemangioma of the rib. Ann Thorac Surg 91: 595-596, 2011.

26. Hao YJ, Yu L, Zhang Y, Wang LM and Li JZ: Surgical treatment of cervical vertebral hemangioma associated with adjacent cervical spondylotic myelopathy. Spine J 13: 1774-1779, 2013.

27. Naama O, Gazzaz M, Akhaddar A, et al: Cavernous hemangioma of the skull: 3 case reports. Surg Neurol 70: 654-659, 2008.

28. Heiss JD, Doppman JL and Oldfield EH: Brief report: relief of spinal cord compression from vertebral hemangioma by intralesional injection of absolute ethanol. N Engl J Med 331: 508-511, 1994

29. Yoo YS, Seo YJ, Noh KC, Patro BP and Kim DY: Arthroscopically assisted anatomical coracoclavicular ligament reconstruction using tendon graft. Int Orthop 35: 1025-1030, 2011.

30. Clements RH, Turnage RB and Tyndal EC: Hemangioma of the rib: a rare diagnosis. Am Surg 64: 1027-1029, 1998.

31. Okumura T, Asamura H, Kondo $\mathrm{H}$, et al: Hemangioma of the rib: a case report. Jpn J Clin Oncol 30: 354-357, 2000.

32. Ulku R, Onat S, Avci A and Ozmen CA: Resection of intercostal hemangioma with involved chest wall and ribs: in an 11-year old girl. Tex Heart Inst J 37: 486-489, 2010.

33. Ly JQ and Sanders TG: Case 65: hemangioma of the chest wall. Radiology 229: 726-729, 2003.

34. Chawla A, Singrakhia M, Maheshwari M, Modi N and Parmar H: Intraosseous hemangioma of the proximal femur: imaging findings. Br J Radiol 79: e64-e66, 2006.

35. Zou F, Dai M, Zhang B and Nie T: Misdiagnosis of a giant intrapelvic schwannoma: A case report. Oncol Lett 6: 1646-1648, 2013. 